\title{
Erratum: Spin flipping with an rf dipole and a full Siberian snake [Phys. Rev. ST Accel. Beams 3, 104001 (2000)]
}

B. B. Blinov, Ya. S. Derbenev, T. Kageya, D. Yu. Kantsyrev, A. D. Krisch, V. S. Morozov, D. W. Sivers, V. K. Wong,

V. A. Anferov, P. Schwandt, and B. von Przewoski

(Published 23 March 2001)

This erratum has an improved rendition of Fig. 4; the error bars were not properly shown in the published version. The content of the article has not changed.

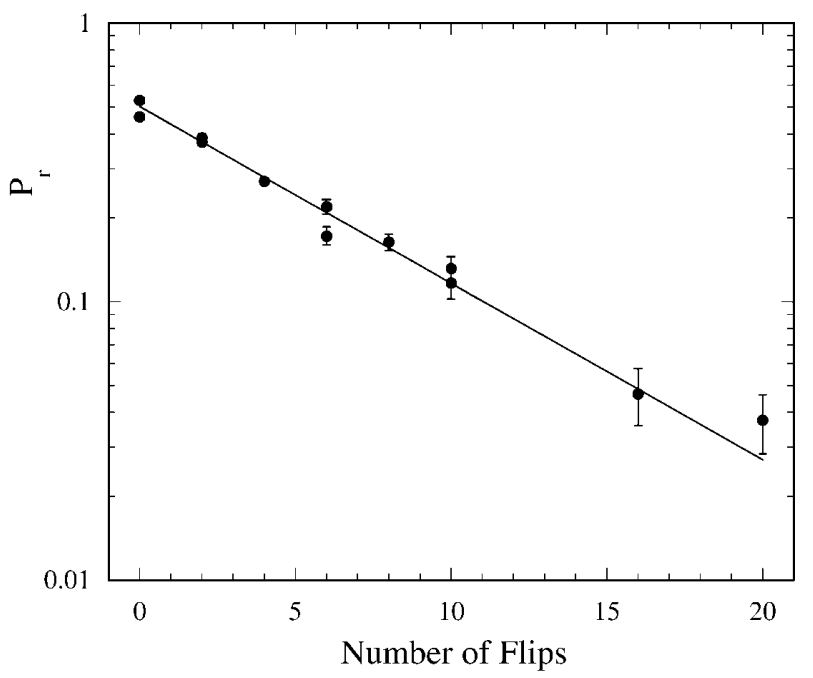

FIG. 4. The measured radial proton polarization at $120 \mathrm{MeV}$ is plotted against the number of spin flips. The rf dipole's frequency ramp time $\Delta t$ was $400 \mathrm{msec}$, its frequency range $\Delta f$ was $10 \mathrm{kHz}$, and its $\int B d l$ was $0.06 \mathrm{Tmm}$. The curve is a fit to the data using Eq. (8). 